\title{
Comparison of retinal and cerebral vasculature in trypsin digest preparations
}

\author{
DAVID G. COGAN ${ }^{1}$ AND TOICHIRO KUWABARA ${ }^{2}$ \\ From the ${ }^{1}$ Neuro-ophthalmology Section and ${ }^{2}$ Laboratory of Vision Research, National Eye Institute, \\ National Institutes of Health, Bethesda, Maryland 20205, USA.
}

SUMMARY The trypsin digestion technique for preparing flat mounts of the retinal capillary system was modified for comparable mounts of the cerebrocortical capillaries. These preparations, supplemented by electron microscopy, showed a basic similarity between the 2 systems, but the cortical capillaries have larger diameters, more abundant collateral channels, and less regularly spaced mural cells (intramural pericytes). The cortical capillaries did not show the right-angle branching of the precapillary arteriole characteristic of some retinal vessels, but they did show in the crotches of the branches an excess basement membrane that was not present in the retinal capillaries.

The architecture of the retinal vasculature has been a dominant interest for many of us these past several decades. Especially noteworthy were the early observations on whole retinal mounts by Michaelson and colleagues utilising benzidine peroxidase ${ }^{1}$ or Indian ink injection, ${ }^{2}$ Friedenwald's use of periodic acid Schiff (PAS) stains, ${ }^{3}$ and Ashton's Indian ink injections. ${ }^{4}$ These illustrated the general design of the retinal circulation, and, although not seriously considered at the time, they invited the question of how circulation could be maintained in a vast capillary bed fed by a single arteriole not much larger than a capillary. Our interest in the retinal vasculature began with the serendipitous observation that partial trypsin digestion of the retina permitted not only preservation of the vascular scaffold but revealed for the first time the distribution and composition of its cellular constitutents. ${ }^{5}$ It was on these cells and their potential for control of the circulation at the capillary level that our attention focused and which we would now like to apply to comparable brain vessels.

The retina is uniquely suitable for the trypsin digest technique: it can be removed with minimal dissection; it contains little trypsin-resistant collagen; and its cells are relatively protected from the digestion by a substantial mucoproteinaceous wall. When subsequently stained by conventional haematoxylin-eosin or PAShaematoxylin, the walls of normal capillaries reveal 2 types of cells, the endothelial cells lining the lumina, and cells encased within the walls of the capillaries. Correspondence to Dr D. G. Cogan.
These latter cells we called mural cells, ${ }^{6}$ but other authors, emphasising their identity with pericytes, have preferred with good reason to call them intramural pericytes. ${ }^{7}$ They have an approximate $1: 1$ frequency with the endothelial cells and appear to be similarly randomly distributed. Histochemical and electron microscopic studies have shown that processes of these cells constitute a substantial part of the walls and embrace the capillaries octopus fashion. There can be little doubt that they play a key role in maintenance of the tone of the retinal capillaries.

A question not previously explored is what relevance these observations on the retina have to other vascular systems, most notably to those of the cortex of the brain. Obviously they have little bearing on capillaries in connective tissue and in certain other tissues where pericytes reside well outside the capillary walls. But cerebral capillaries, like those in the retina, are situated in a neurono-glial matrix and have similarly thick capillary walls. It seemed worthwhile, therefore, to attempt comparable trypsin digest preparations of cortical capillaries.

The cortex of the brain entails several disadvantages by comparison with the retina. In the first place the capillary and paracapillary vessels arise from the pia as multiple branches rather than as discrete arteriolovenular circuits. Secondly, the cortex contains connective tissue and lipids which impair the trypsin digestion. Nevertheless modifications of the technique have enabled preparations that warrant a preliminary report. 


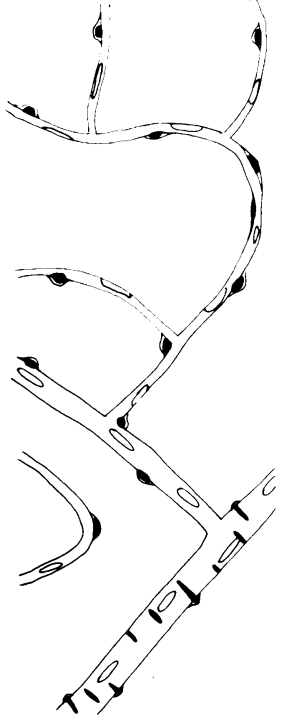

Retina

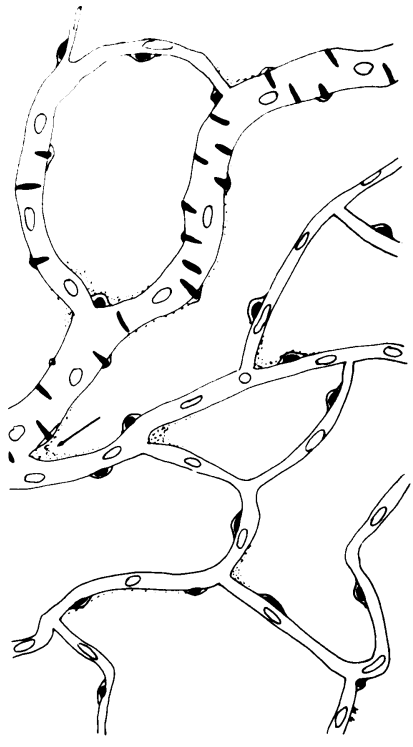

Brain
$100 \mu \mathrm{m}$

Fig. 1 Diagram illustrating typical portions of retinal and cerebral cortical vasculature. Feeding vessels for retinal capillaries often come off at right angles from the parent arteriole and then branch successively with uniform bores. Brain capillaries by contrast characteristically arise exclusively by dichotomous branching with frequent feedback loops and nonuniform bores. The cerebral capillaries also differ in showing membranous material and occasional entrapped cells in the crotch of capillary and arteriolar branches (arrow).

\section{Material and methods}

Small pieces of formalin-fixed brain cortex (approximately $1 \times 1 \times 0.3 \mathrm{~cm})$ were excised and the pia stripped off. The pieces were then washed in water and dehydrated in ascending strengths of alcohol ( 30 minutes in each of 75,85 , and $90 \%$ followed by immersion in absolute alcohol for one hour). They were further delipidised in a mixture of 4 parts of methanol and 1 part chloroform. The extraction was continued for 24 hours with frequent shaking. The tissue was then brought back to water by passing through descending alcohols. The blood vessels were then isolated by digestion of the tissue in $3 \%$ trypsin (Difco 1:250) for 1-3 hours. This solution was made up freshly in $0 \cdot 15 \mathrm{M}$ tris buffer $(\mathrm{pH} 7 \cdot 8)$. The digestion resulted in fragments of tissue measuring approximately $1-3 \mathrm{~mm}^{3}$. These fragments containing samples of blood vessels were then further digested in fresh trypsin solution with intermittent ultrasonic vibration. The resultant turbid solution was then suspended in water and the fragments of blood vessels visualised by illumination from the side with collimmated light. The vessels, often characterised by Y branches, stood out against a dark background, but large segments were rarely obtained. The extraneous debris was cleared away and the samples of blood vessels were mounted on glass slides. The preferred staining was the use of PAS and haematoxylin.

\section{Results}

The trypsin digest preparations of the cortex are possible but less satisfactory than those of the retina. Whole cortical mounts do not enable visualisation of intact arteriolovenular circuits. One must deal with small segments of the circuits. Moreover, the intercapillary connective tissue and lipid in the cortex interfere with clean digest preparations. Nevertheless, one may obtain mounts which, together with electron microscopic observations, permit a measure of comparison and the following conclusions (Fig. 1).

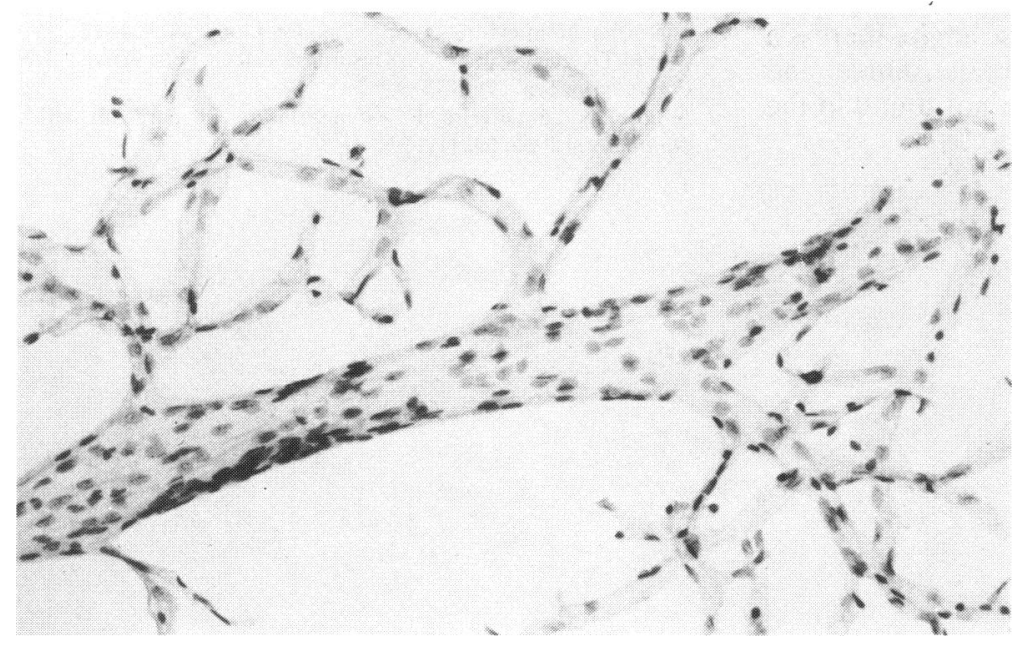

Fig. 2 Trypsin digest preparation of cerebral, cortical vasculature showing: (1) dark staining round nuclei of mural cells, and pale staining ellipsoid cells of endothelium; (2) nonuniform bore of capillaries; (3) exclusively obtuse branching of capillaries; and (4) feed-back loops. (Haematoxylin$P A S, \times 107)$. 


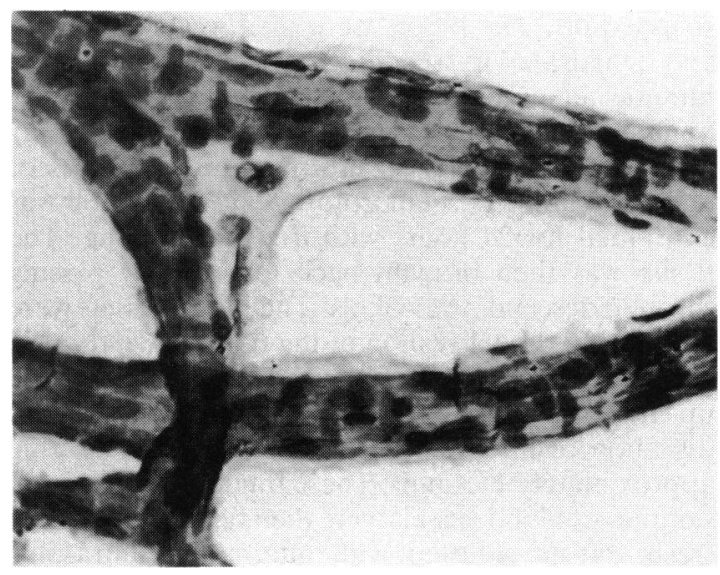

Fig. 3 Trypsin digest preparation of cerebral cortical vessels showing membranous substance with contained cells in crotch of a branching arteriole. (Haematoxylin-PAS, $\times 236$ ).

POINTS OF SIMILARITY

(1) Both retinal and cortical capillaries have unusually substantial basement membranes (as compared with connective tissue capillaries). This implies a limited capacity for expansion.

(2) Both retinal and cortical capillaries have mural cells that constitute an intrinsic part of the wall of the capillaries (Fig. 2). It is assumed that these cells have functions analogous to pericytes in other capillaries but because of their intramural position have additional pathological significance.

POINTS OF DISSIMILARITY

(1) The capillaries of the cortex have diameters 2-5 times that of the retina and are less uniformly bored.

(2) The cortical vessels branch dichotomously at obtuse angles without the right-angle branching so characteristic of the retinal vessels in the posterior part of the eye. In the crotch of these angles there is a tissue made up of basement membrane, fibres, and sometimes cells (Fig. 3). These are not found in the retinal vessel preparations.
(3) The cortical capillaries form abundant collateral loops, rarely if ever seen in normal retinal vessels.

(4) The mural cells are less frequent and less regularly spaced in cortical capillaries than in retinal capillaries.

RELEVANCE TO PATHOLOGY

One of the cases from which the cerebral capillaries were isolated was a patient with severe diabetic retinopathy. This case did not show the loss of the mural cells so characteristic of diabetic retinopathy. This observation on a single case needs to be evaluated in further studies.

\section{Discussion}

The fact that retinal and cortical capillaries have mural cells constituting part of their walls suggests a common property of tonic control related to their thick walls. It might also suggest a shared vulnerability to disease processes affecting these cells. Yet along with certain other differences cited in the foregoing test, the mural cells are less frequent and less regularly distributed in the cortical capillaries. The implications of these differences are beyond the scope of this preliminary report.

The authors acknowledge the valuable technical assistance of Mary Alice Crawford.

\section{References}

1 Michaelson IC, Campbell ACP. The anatomy of the finer retinal vessels. Trans Ophthalmol Soc UK 1940; 60: 71-112.

2 Michaelson IC, Steedman HF. Injection of the retinal vascular system in enucleated eyes. Br J Ophthalmol 1949; 33: 376-9.

3 Friedenwald JS. A new approach to some problems of retinal vascular disease. Am J Ophthalmol 1949; 32: 487-98.

4 Ashton N. Injection of retinal vascular system in diabetic retinopathy. Br J Ophthalmol 1950; 34: 38-41.

5 Kuwabara T, Cogan DG. Studies of retinal vascular patterns, Pt. 1. Normal architecture. Arch Ophthalmol 1960; 64: 904-11.

6 Cogan DG, Kuwabara T. The mural cell in perspective. Arch Ophthalmol 1967; 78: 133-9.

7 Ashton N, deOliveira F. Nomenclature of pericytes. Br J Ophthalmol 1966; 50: 119-23. 